\title{
Enhanced type inference for binding-time analysis
}

\author{
Mátyás SZOKOLI \\ Eötvös Loránd University \\ Budapest, Hungary \\ email: vyu4a5@inf.elte.hu
}

\author{
Attila KISS \\ J. Selye University \\ Komárno, Slovakia \\ email: kissae@ujs.sk
}

\begin{abstract}
In this paper we will be taking a look at type inference and its uses for binding-time analysis, dynamic typing and better error messages. We will propose a new binding-time analysis algorithm $\mathcal{B}$, which is a modification of an already existing algorithm by Gomard [4], and discuss the speed difference.
\end{abstract}

\section{Introduction}

Binding-time analysis (BTA) is used to guide partial evaluation, which is an optimization technique that from an input program produces an output program behaving in the same way, with the output having faster run time. We divide the program into separate parts based on two or more stages. In the case of two stages these are the static and dynamic parts. The compiler can perform the static computations in compile time, yielding a more efficient residual program. This analysis can be done from multiple angles and approaches: through abstract interpretation, type inference, temporal logic and type-based search. One such method developed by Gomard uses the widely-known W type inference algorithm. We replace this inference algorithm by M. We show that this modified algorithm is correct, and is faster then using $\mathrm{W}$ in many cases.

Computing Classification System 1998: D.1.1, F.4.1, F.3.2, F.3.3

Mathematics Subject Classification 2010: 68N18, 68N15, 03 B38

Key words and phrases: type inference, dynamic typing, binding-time analysis, partial evaluation, functional languages 


\section{Related works}

The value of type systems for binding-time analysis was found early, both Nielson [10] and Gomard [3] formalized it as a type inference problem for the two-level typed $\lambda$-calculus. Algorithm $\mathcal{W}$ was already used by both Gomard and Nielsen for their respective $\lambda$-calculus variants. For a detailed look into types and type systems beyond what is included in this paper, see the works of Benjamin C. Pierce $[15,16]$.

Tim Sheard and Nathan Linger introduced a search-based method to perform BTA [11], and they implemented it for MetaML. MetaML already had manual staging annotations, and they integrated automatic BTA to get a unified system to get the advantages from both manual and automated annotations, as they felt that both ways are beneficial to the programmer. Their proposal integrates automatic BTA as a part of the language, not an external tool.

Takuma Murakam et al. also worked with binding-time analysis based on MetaML [9], but they solved it using the maximum marking problem, and used the program transformation optimization theorem for maximum marking to achieve an effective algorithm. This also had the advantage of having a formal computational complexity and guaranteeing the optimality of the solutions based on a given weight function. They also allow the user to define their own weight measurements.

Kenichi Asai introduced binding-time analysis to MetaOCaml [1], a staged language. He relates the 2 -level $\lambda$-calculus with staged $\lambda$-calculus. The advantage of his approach is that the optimal binding times can be found easier in the 2-level $\lambda$-calculus, avoiding searching or complex constraint solving. This is in opposition to Sheard and Linger, who also considered multiple stages

and polymorphism. Kenichi Asai thinks it is not clear if his approach supports these two features.

These works show that even the type-theoretic viewpoint has several separate solutions. Our contribution concerns the use of classic type inference algorithms for this purpose.

\section{Additional uses of type inference}

\subsection{Binding-time analysis}

Binding-time analysis is used to reason about the availability of the data, that is, which parts of a program we can evaluate at compile time. Guided 
by this information, we can produce a residual program, which only contains computations that cannot be executed at compile time. We will be using the terms "dynamic", "late-bound" interchangeably.

Binding-time analysis aims to produce a minimal completion - the least amount of program parts should be dynamic. Different solutions were proposed for this problem, one of them being the use of type inference and of two-level expressions.

We can view this analysis as a type inference problem, where we are trying to produce a completion which satisfies some typing rules.

However, as mentioned, these can be also used for other problems, namely type checking dynamic types, and providing more comprehensive type errors in statically typed languages.

\subsection{Dynamic typing}

We can split functional programming languages into two separate groups, statically typed ones like Haskell, ML and dynamically typed languages, e. g. Lisp, Scheme, Clojure and Erlang. In statically typed languages types are known at compile-time. This means that we don't need typechecking in the runtime, which can be a performance bonus. This can also replace some basic tests, as it narrows down the domain of possible input values. In a rich type system types can also act as documentation, detailing the computation itself.

On the other hand dynamic languages can offer great flexibility at the cost of some performance. In the former languages through many so-called typechecking algorithms we have assurances, that no type errors will arise at runtime. This analysis can be done in compile time. In dynamically typed languages we must keep track of types in the runtime, and type errors often only can be found at runtime. However there exist many separate approaches to provide as much type safety as we can while keeping dynamic typing.

Staged type inference is run multiple times. One possibility is to separate dynamic and static parts, and run checks on them in compile time and runtime.

Success typing finds definite type clashes without changing the source language fundamentally, and was implemented for Erlang in the popular static analysis tool Dialyzer [7].

We will present an approach in this paper, which finds all possible sources of type errors in the program, and underline them. Later, we will discuss ways we can improve the performance and enrich the languages we can examine, and other possible uses. Our algorithm, $\mathcal{B}$ is based on the one presented by Gomard [4]. 


\section{Overview}

These problems are quite similar: we are searching for points where the expression is not well-formed in regards to some predefined rules. In the former it means that these conflicting parts cannot be executed at compile time, and in the latter that we don't know the types statically.

Viewed from a type-theoretic angle these can be handled as type inference problems: inferring a completion of a term with annotations so that it is welltyped in regards to some type rules. Gomard proved that for every term of his two-level $\lambda$-calculus exists a completion that minimizes late binding.

Figures 1 and 2 show such a system. This is almost directly lifted from Gomard [4], the difference being that we use $\Lambda$ to mean the type Untyped for the sake of brevity.

For our purposes we will be using lambda calculus with constants, conditional branching, and an explicit fixpoint-operator. We will be using a Currystyle type system. There are several implications of this: firstly, we can view untyped programs as potentially typed programs without annotations, and the expressions whose types can be inferred as well-typed.

Naturally, this technique can be used with other type systems that have decidable type inference capabilities. We will be using a modified version of Algorithm $\mathcal{M}(\underline{\mathcal{M}})$ instead of $\mathcal{W} . \mathcal{M}$ is also sound and complete, and was shown to always find type errors earlier than $\mathcal{W}[6]$. We will examine how this behaviour is affected by the modified type system.

The extended lambda calculus with annotations is defined by the following production:

$$
\begin{aligned}
e::= & x\left|\lambda x \cdot e^{\prime}\right| e^{\prime} @ e^{\prime \prime} \mid \text { if } e^{\prime} e^{\prime \prime} e^{\prime \prime \prime} \mid \text { fix e } \mid \text { const }_{n} \text { base-value } n \mid \\
& \underline{x}\left|\underline{\lambda} x \cdot e^{\prime}\right| e^{\prime} \underline{@} e^{\prime \prime}\left|\underline{\text { if }} e^{\prime} e^{\prime \prime} e^{\prime \prime \prime}\right| \underline{\text { fix } e \mid \underline{\text { const }}_{n} \text { base-value }}{ }_{n}
\end{aligned}
$$

where $\mathrm{x}$ ranges over a class of variables, $\mathrm{n}$ is a natural number, base-value ${ }_{n}$ is member of the set of curried n-ary base functions on first order values. and $f i x$ is a fix-point operator.

Binding time information is represented by types. Our types $\tau$ are generated like so:

$$
\tau::=\text { Base }_{\omega}|\Lambda| \alpha \mid \tau^{\prime} \rightarrow_{\omega} \tau^{\prime \prime}
$$

Base is a type constant, it represents concrete static values - values known at compile-time. $\Lambda$ stands for values not known at compile-time, or in the second use case it means an unknown type at compile-time. $\alpha$ denotes the set of type variables, and $\tau_{1} \rightarrow_{\omega} \tau_{2}$ is a function type. $\omega$ is a sequence describing 
where the type was inferred in the examined expression. For instance in the expression $(\lambda x . x) @$ const the right hand side's type is Base Arg::* $_{\text {. }}$

$$
\begin{aligned}
& \omega::=* \mid \text { Fun }:: \omega \mid \text { Arg }:: \omega \mid \text { Body }:: \omega \mid \text { FixBody }:: \omega \mid \\
& \quad \text { Cond }:: \omega \mid \text { Then }:: \omega \mid \text { Else }:: \omega
\end{aligned}
$$

\begin{tabular}{|c|c|}
\hline$\frac{\Gamma \vdash e: \tau \rightarrow \tau}{\Gamma \vdash \text { fix } e: \tau}$ & [FIX1] \\
\hline$\frac{\Gamma \vdash e_{1}: \text { Base } \Gamma \vdash e_{2}: \tau \quad \Gamma \vdash e_{3}: \tau}{\Gamma \vdash \text { if } e_{1} e_{2} e_{3}: \tau}$ & [IF1] \\
\hline$\frac{\Gamma \vdash e_{1}: \tau_{2} \rightarrow \tau_{1} \quad \Gamma \vdash e_{2}: \tau_{2}}{\Gamma \vdash e_{1} @ e_{2}: \tau_{1}}$ & [APP1] \\
\hline$\frac{\Gamma, x: \tau_{1} \vdash e: \tau_{2}}{\Gamma \vdash \lambda x . e: \tau_{1} \rightarrow \tau_{2}}$ & [ABS1] \\
\hline$\frac{\Gamma(x)=\tau}{\Gamma}$ & [VAR1] \\
\hline $\begin{array}{c}\overline{\Gamma \vdash x: \tau} \\
\Gamma \vdash \text { const }: \text { Base } \\
\Gamma \vdash \text { const }_{1}: \text { Base } \rightarrow \text { Base }\end{array}$ & $\begin{array}{l}{[\mathrm{CON} 1]} \\
{[\mathrm{CONFN} 1]}\end{array}$ \\
\hline
\end{tabular}

Figure 1: Basic expressions

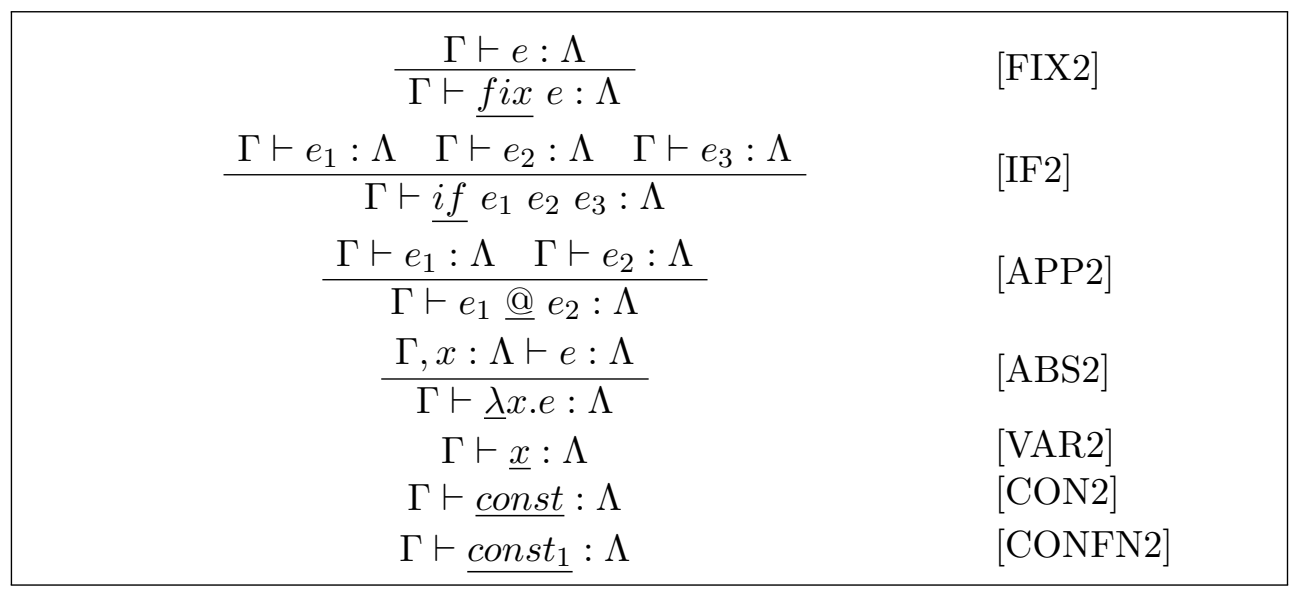

Figure 2: Underlined expressions 
Binding-time information is represented syntactically: a late-bound/dynamic operator is different from it's matching basic version by being underlined. A $\lambda$-term with these annotations is called an annotated $\lambda$-term. If a term satisfies the inference rules in Figures 2 and 1 we also call it "well-annotated".

We call an untyped $\lambda$-term the erasure of an annotated $\lambda$-term $e$ if the untyped term has no operator and type annotations, but otherwise is identical to $e$. A completion of a non-annotated term $e$ with reference to some typing assumptions $\mathcal{A}$ is a well-annotated term w.r.t $\mathcal{A}$ with the erasure $e$.

We can get a trivial completion for every untyped $\lambda$-term by underlining all of the subexpressions. A minimal completion is a completion with the minimal amount of late-binding operators. Gomard presented a modified $\mathcal{W}(\underline{\mathcal{W}})$ algorithm, and conjectures that using it we can compute minimal completions, which we will compare to our $\underline{\mathcal{M}}$ algorithm.

We give a possible definition of Gomard's algorithm $\left(\mathcal{B}^{\prime}\right)$. Then we will detail ours $(\mathcal{B})$.

$$
\begin{gathered}
\mathcal{B}^{\prime}(e, \Gamma)=\left\{\begin{array}{l}
\mathcal{B}^{\prime}(U(e, \omega), \Gamma), \text { if } \underline{\mathcal{W}}(e, \Gamma, *)=F A I L(\omega) \\
\text { e otherwise }
\end{array}\right. \\
\mathcal{B}(e, \Gamma)=\left\{\begin{array}{l}
\mathcal{B}(U(e, \omega), \Gamma), \text { if } \underline{\mathcal{M}}(\Gamma, e, \beta, *)=F A I L(\omega), \text { new } \beta \\
\text { e otherwise }
\end{array}\right.
\end{gathered}
$$

We provide no definition for the $U$ function, which has the sole purpose of underlining the operator in the $e$ parameter which is pointed to by the $\omega$ occurrence parameter. Both $\underline{\mathcal{M}}$ and $\underline{\mathcal{W}}$ are extended to support underlined operators. The $\mathcal{M}$ algorithm is detailed in Fig. 4.

To summarize both $\mathcal{B}$ and $\mathcal{B}^{\prime}$ : both algorithms run their respective type inference algorithms first, to check if the $e$ input is well typed w.r.t the $\Gamma$ type environment. If the type of $e$ is inferred successfully, then it is finished. If $\underline{\mathcal{M}}$ or $\underline{\mathcal{W}}$ announce failure, we iterate once more, but with a modified $e$, which has the offending operator underlined.

Theorem 1 (Oukseh Lee, Kwangkeun Yi [6]) Let $\Gamma$ be a type environment, e an expression and $\beta$ a new type variable. Then

$$
|\llbracket \mathcal{M}(\Gamma, e, \beta) \rrbracket| \leq|\llbracket \mathcal{W}(\Gamma, e) \rrbracket|
$$

$\mathcal{M}$ 's call string is shorter or equal to $\mathcal{W}$ 's. Put in other words, the first type inference algorithm makes fewer or the same amount of recursive calls. We will see later what this means in practice 
$\mathcal{W}$ is bottom-up, and $\mathcal{M}$ is top-down, they process expressions in a different order. The former infers the type of every subexpression, and then tries to unify them, while the latter tries to check each subexpression after another with a constraint, which will be unified at the "leaf nodes". This means that it often catches errors earlier that violate the constraint. The main difference can be seen with $e_{1} @ e_{2}$ : it is possible, that a not well-typed subexpression is typechecked by $\mathcal{W}$ before it gets into conflict at the application. In the cases where the left-hand side of the application is not a valid function, $\mathcal{M}$ only has to examine it, while $\mathcal{W}$ has to check both and then unify.

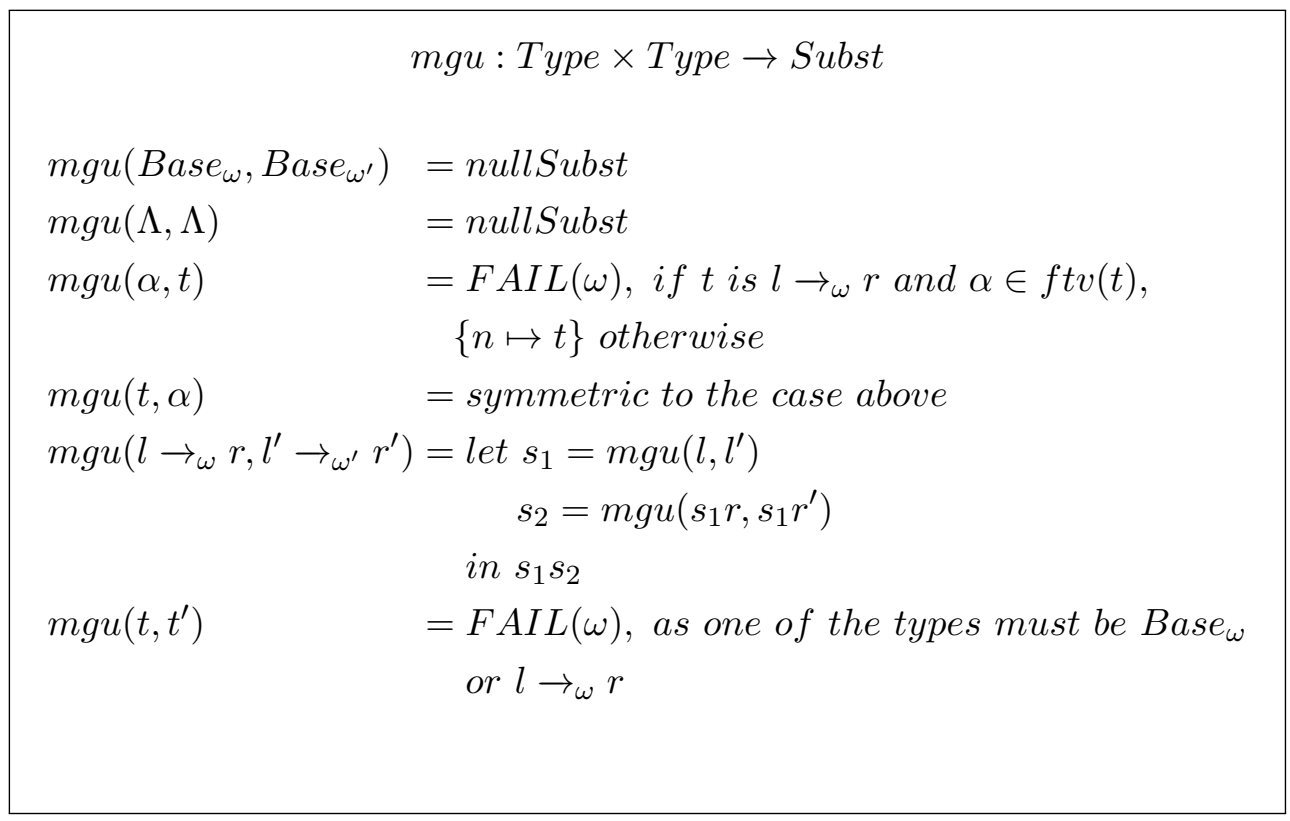

Figure 3: The unification used. $t, t^{\prime}, l, l^{\prime}, r, r^{\prime} \in \tau$ are arbitrary types, $\alpha$ is a type variable. 


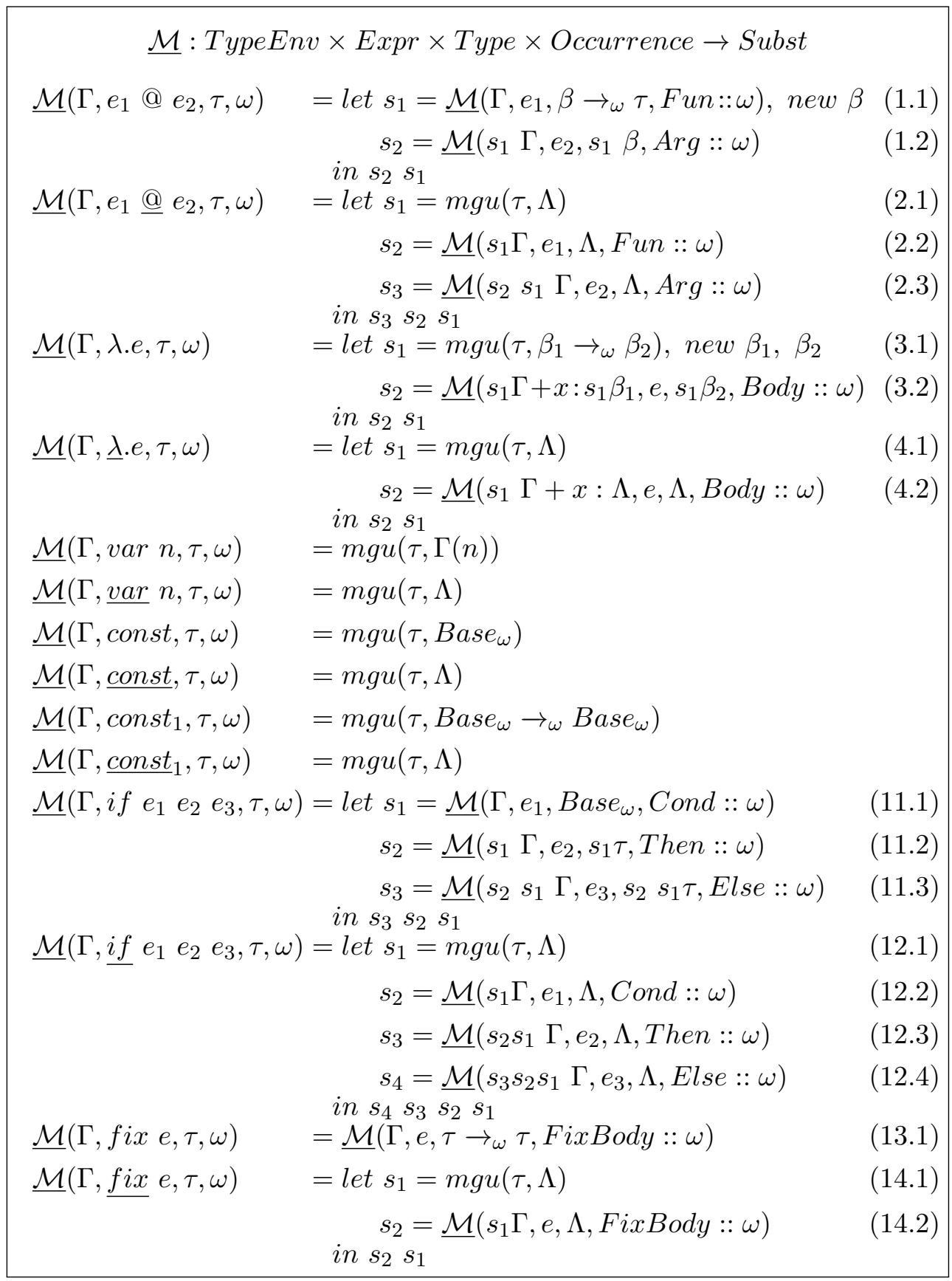

Figure 4: The modified M algorithm 


\section{Proof of correctness}

Lemma 2 (Damas and Milner) If $S$ is a substitution and $\Gamma \vdash e: \tau$, then $S \Gamma \vdash e: S \tau[8]$

Lemma $3(\underline{\mathcal{M}}$ is sound) Let e be an expression and $\Gamma$ a type environment. If there exist a type $\tau$ such that $\underline{\mathcal{M}}(\Gamma, e, \tau, *)=S$, then $S \Gamma \vdash e: S \tau$.

This section heavily builds upon the work of Oukseh Lee and Kwangkeun Yi [6]. The only differences are that we have a new constant type, $\Lambda$, and that we are not using polymorphism in this instance. We will use structural induction on $e$.

Proof.

- case const: $S \tau=S$ Base $_{\omega}=$ Base $_{\omega}$. So $S \Gamma \vdash$ const : $S \tau$ by [CON1].

- case const: $S \tau=S \Lambda=\Lambda$. So $S \Gamma \vdash \underline{\text { const }}: S \tau$ by [CON2].

- case const $_{1}: S \tau=S\left(\right.$ Base $_{\omega} \rightarrow$ Base $\left._{\omega^{\prime}}\right)=$ Base $_{\omega} \rightarrow$ Base $_{\omega^{\prime}}$. So $S \Gamma \vdash$ const $_{1}: S \tau$ by [CONFN1].

- case $\underline{\text { const }}_{1}: S \tau=S \Lambda=\Lambda$. So $S \Gamma \vdash \underline{\text { const }}_{1}: S \tau$ by [CONFN2].

- case $x: S \tau=S \Gamma(x)$. So $S \Gamma \vdash x: S \tau$ by [VAR1].

- case $\underline{x}: S \tau=S \Lambda=\Lambda$. So $S \Gamma \vdash \underline{x}: S \tau$ by [VAR2].

- case $\lambda x . e$ :

1. By induction, (3.2) implies

$$
S_{2} S_{1} \Gamma+x: S_{2} S_{1} \beta_{1} \vdash e: S_{2} S_{1} \beta_{2} .
$$

2. By [ABS1] $S_{2} S_{1} \Gamma \vdash \lambda x . e: S_{2} S_{1} \beta_{1} \rightarrow S_{2} S_{1} \beta_{2}$; which is, by (3.1)

$$
S_{2} S_{1} \Gamma \vdash \lambda x . e: S_{2} S_{1} \tau \text {. }
$$

- case $\underline{\lambda} x . e$ :

1. By induction, (4.2) implies

$$
S_{2} S_{1} \Gamma+x: S_{2} \Lambda \vdash e: S_{2} \Lambda \text {. So } S_{2} S_{1} \Gamma+x: \Lambda \vdash e: \Lambda \text {. }
$$


2. By [ABS2] $S_{2} S_{1} \Gamma \vdash \underline{\lambda x} . e: S_{2} S_{1} \Lambda$; which is, by (4.1)

$$
S_{2} S_{1} \Gamma \vdash \underline{\lambda} x . e: S_{2} S_{1} \tau .
$$

- case $e_{1} @ e_{2}$ :

1. By induction, (1.1) implies $S_{1} \Gamma \vdash e_{1}: S_{1}(\beta \rightarrow \tau)$. By Lemma 2, we can apply $S_{2}$ to both sides.

$$
S_{2} S_{1} \Gamma \vdash e_{1}: S_{2} S_{1} \beta \rightarrow S_{2} S_{1} \tau
$$

2. By induction, (1.2) implies

$$
S_{2} S_{1} \Gamma \vdash e_{2}: S_{2} S_{1} \beta .
$$

3. By $[\mathrm{APP} 1]$,

$$
S_{2} S_{1} \Gamma \vdash e_{1} @ e_{2}: S_{2} S_{1} \tau
$$

- case $e_{1} @ e_{2}$ :

1. By induction, (2.2) implies $S_{2} S_{1} \Gamma \vdash e_{1}: S_{2} S_{1} \Lambda$. By Lemma 2, we can apply $S_{3}$ to both sides.

$$
S_{3} S_{2} S_{1} \Gamma \vdash e_{1}: S_{3} S_{2} S_{1} \Lambda \text {. So } S_{3} S_{2} S_{1} \Gamma \vdash e_{1}: \Lambda .
$$

2. By induction, (2.3) implies

$$
S_{3} S_{2} S_{1} \Gamma \vdash e_{2}: S_{3} \Lambda \text {. So } S_{3} S_{2} S_{1} \Gamma \vdash e_{2}: \Lambda \text {. }
$$

3. By [APP2] $S_{3} S_{2} S_{1} \Gamma \vdash e_{1} \underline{@} e_{2}: \Lambda$. So $S_{3} S_{2} S_{1} \Gamma \vdash e_{1} \underline{@} e_{2}: S_{3} S_{2} S_{1} \Lambda$. That is, by (2.1),

$$
S_{3} S_{2} S_{1} \Gamma \vdash e_{1} @ e_{2}: S_{3} S_{2} S_{1} \tau .
$$

- case if $e_{1} e_{2} e_{3}$ :

1. By induction, (11.1) implies $S_{1} \Gamma \vdash e_{1}: S_{1}$ Base. By Lemma 2, we can apply $S_{2}$ and $S_{3}$ to both sides:

$$
S_{3} S_{2} S_{1} \Gamma \vdash e_{1}: S_{3} S_{2} S_{1} \text { Base. }
$$

2. By induction, (11.2) implies $S_{2} S_{1} \Gamma \vdash e_{2}: S_{2} S_{1} \tau$. By Lemma 2, we can apply $s_{3}$ to both sides:

$$
S_{3} S_{2} S_{1} \Gamma \vdash e_{2}: S_{3} S_{2} S_{1} \tau
$$


3. By induction, (11.3) implies

$$
S_{3} S_{2} S_{1} \Gamma \vdash e_{3}: S_{3} S_{2} S_{1} \tau .
$$

4. By [IF1],

$$
S_{3} S_{2} S_{1} \Gamma \vdash \text { if } e_{1} e_{2} e_{3}: S_{3} S_{2} S_{1} \tau
$$

- case if $e_{1} e_{2} e_{3}$ :

1. By induction, (12.2) implies $S_{2} S_{1} \Gamma \vdash e_{1}: S_{2} \Lambda$. By Lemma 2, we can apply $S_{3}$ and $S_{4}$ to both sides:

$$
S_{4} S_{3} S_{2} S_{1} \Gamma \vdash e_{1}: S_{4} S_{3} S_{1} \Lambda \text {. So } S_{4} S_{3} S_{2} S_{1} \Gamma \vdash e_{1}: \Lambda \text {. }
$$

2. By induction, (12.3) implies $S_{3} S_{2} S_{1} \Gamma \vdash e_{2}: S_{3} \Lambda$. By Lemma 2, we can apply $S_{4}$ to both sides:

$$
S_{4} S_{3} S_{2} S_{1} \Gamma \vdash e_{2}: S_{4} S_{3} \Lambda \text {. So } S_{4} S_{3} S_{2} S_{1} \Gamma \vdash e_{2}: \Lambda \text {. }
$$

3. By induction, (12.4) implies

$$
S_{4} S_{3} S_{2} S_{1} \Gamma \vdash e_{3}: S_{4} \Lambda \text {. So } S_{4} S_{3} S_{2} S_{1} \Gamma \vdash e_{3}: \Lambda \text {. }
$$

4. By [IF2] $S_{4} S_{3} S_{2} S_{1} \Gamma \vdash \underline{i f} e_{1} e_{2} e_{3}: \Lambda$. So $S_{4} S_{3} S_{2} S_{1} \Gamma \vdash \underline{i f} e_{1} e_{2} e_{3}$ : $S_{4} S_{3} S_{2} S_{1} \Lambda$, that is, by (12.1)

$$
S_{4} S_{3} S_{2} S_{1} \Gamma \vdash \underline{\text { if }} e_{1} e_{2} e_{3}: S_{4} S_{3} S_{2} S_{1} \tau
$$

- case fix e:

1. By induction, (13.1) implies $S_{1} \Gamma \vdash e: S_{1}(\tau \rightarrow \tau)$, which is by definition

$$
S_{1} \Gamma \vdash e: S_{1} \tau \rightarrow S_{1} \tau
$$

2. By [FIX1],

$$
S_{1} \Gamma \vdash \text { fix e }: S_{1} \tau \text {. }
$$

- case $\underline{\text { fix }}$ e:

1. By induction, (14.2) implies

$$
S_{2} S_{1} \Gamma \vdash e: S_{2} \Lambda \text {. So } S_{2} S_{1} \Gamma \vdash e: \Lambda \text {. }
$$


2. By [FIX2] $S_{2} S_{1} \Gamma \vdash \underline{\text { fix }} e: \Lambda$. So $S_{2} S_{1} \Gamma \vdash \underline{\text { fix }} e: S_{2} S_{1} \Lambda$, that is, by (14.1)

$$
S_{2} S_{1} \Gamma \vdash \underline{\text { fix }} e: S_{2} S_{1} \tau \text {. }
$$

Lemma 4 No operator is underlined twice.

Proof. To be subject to underlining, an operator has to have their occurrence in the exception raised by the $m g u$ algorithm. This can only happen if the occurrence is in one of $m g u$ 's parameters. There are two ways some $o$ operator's occurrence can be one of the the parameters of an mgu call:

- This can happen directly if we call mgu with $o$ 's occurence when $o$ is processed (see const).

- If we make a recursive call to $\underline{\mathcal{M}}$, where the type parameter has $o$ 's occurrence (see 1.1), which is unified later.

If we take a look at $\underline{\mathcal{M}}$ 's branches where the expression parameter is underlined, we can see that neither of these conditions are met.

Theorem 5 (The $\mathcal{B}$ algorithm is correct) Let e be some starting lambda expression and $\Gamma$ a starting type environment. $\mathcal{B}(e, \Gamma)$ completes in finite steps, and for the expression $e^{\prime}$, where $\mathcal{B}(e, \Gamma)=e^{\prime}$ :

- There exist some $\tau$ type and $S$ substitution, such that $S \Gamma \vdash e^{\prime}: S p$.

- $e$ is the erasure of $e^{\prime}$.

Proof. Let $n(e) \in \mathbb{N}$ be the number of underlined operators in $e$, and $s(e) \in \mathbb{N}$ be the total number of operators in the expression in $e$, underlined or otherwise. It is trivial, that for every $e \lambda$-term $n(e) \leq s(e)$. On each recursive step, based upon $\underline{\mathcal{M}}$ 's result either:

- $\underline{\mathcal{M}}(\Gamma, e, \tau, *)=F A I L(\omega)$ for some new $\tau$. We underline the indicated operator, $e^{\prime}=U(e, \omega)$. Because of Lemma 4 we can say that this is a new underlining, so $n\left(e^{\prime}\right)=n(e)+1$. We continue the recursion with $e^{\prime}$.

- If $\underline{\mathcal{M}}(\Gamma, e, \tau, *)=S$, then according to Lemma 3 we get $S \Gamma \vdash e: S p$. This means that the expression $e$ in the environment $\Gamma$ is well typed w.r.t. the type inference system presented in figures 1 and 2 , so $e$ is correctly annotated. 
If follows, that if $\mathcal{B}$ stops, the first case is true, so we have the correct output. This means that the only way $\mathcal{B}$ be incorrect, is if it doesn't halt. But because $n(e)$ grows by one between each recursive call, we must reach the state where $n(e)=s(e)$. If $\underline{\mathcal{M}}(\Gamma, e, \tau, *)=F A I L(\omega)$, then we must underline a new nonunderlined operator, but because $n(e)=s(e)$, there is no operator that is lined.

We defined $U$ as a function that only underlines operators. Between every recursion we modify the expression in no other way, meaning that the output expression's erasure is the initial input expression.

\section{$6 \quad$ Examples}

In this section we will be taking a look at a few examples, and how our algorithm's behaviour differs from that of Gomard's.

\subsection{Simple expression}

Let us examine the expression const @ const, where const-s are some arbitrary constants. The current analysis doesn't differentiate between constant types, so this could consist of any two expressions

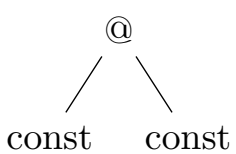
with such types like Int, Bool. $\underline{\mathcal{W}}$ infers the type of the left-hand side, then the right-hand side, then fails at unification:

$$
t_{\text {left }}=t_{\text {right }} \rightarrow T
$$

(where $\mathrm{T}$ is a new type variable), because $t_{\text {left }}$ and $t_{\text {right }}$ are both Base. In case of a unification failure, the occurrence of one of the types is sent, which in our implementation's case is that of the left. We can see that on each round we must check both subexpressions.

$\mathcal{M}$ however finds this problem one step earlier. According to the algorithm above, it checks if the left subexpression has a function type, and here it fails, because the type found is Base $_{\omega}$ and fails with the same occurrence as earlier.

After underlining the expression we try again. We fail at the same place, but this time it is with the constraint

$$
\Lambda=\tau \rightarrow T
$$

We have the occurrence of the equation's right side as error message. The selector in question is empty, because the application is the root of the whole 
expression. This means that we will underline the application. Upon restarting, we branch again at the underlined application. This time left-hand side checks out, because we both expect and get $\Lambda$. There is conflict on the right however: we expect $\Lambda$, but have Base. The second const gets underlined, then we restart the algorithm again, which is finally successful.

\subsection{Y combinator}

For this second example we will take a look at the Y combinator. This fixpointcombinator is not well-typed, so we will need to underline the problem areas to conform to the type rules. We have numbered these in the figure for an easier understanding. The numbering is according to the order in which $\underline{\mathcal{W}}$ finds them. Both algorithms find 1. first. Here $\underline{\mathcal{M}}$ examines the same number of subexpressions, we don't gain any advantage at the application. This is because the left-hand side's type is a type variable that hasn't had any constraints or sub-

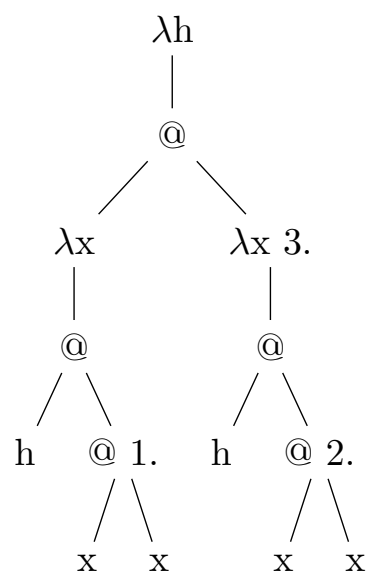
stitutions placed upon it, so it can be unified with a function type. ${ }^{1}$ Conflict only arises after visiting the other side with

$$
T=\tau \rightarrow T
$$

The rest of the errors are found earlier. In these two cases the different ways of inferring application types do come in to play. To summarize, the main cause of difference is that at applications $\underline{\mathcal{W}}$ has to infer both children, while in some cases $\underline{\mathcal{M}}$ can do it by only one.

\section{Implementation and random testing}

Random testing compiler optimizations have become more common practice in the last decades as computing power grew and the necessary frameworks and algorithms were established. We will be using the package QuickCheck [2]

\footnotetext{
${ }^{1}$ This is why $\lambda x . x @ x$ takes the same number of visited nodes despite being similar to the first example.
} 


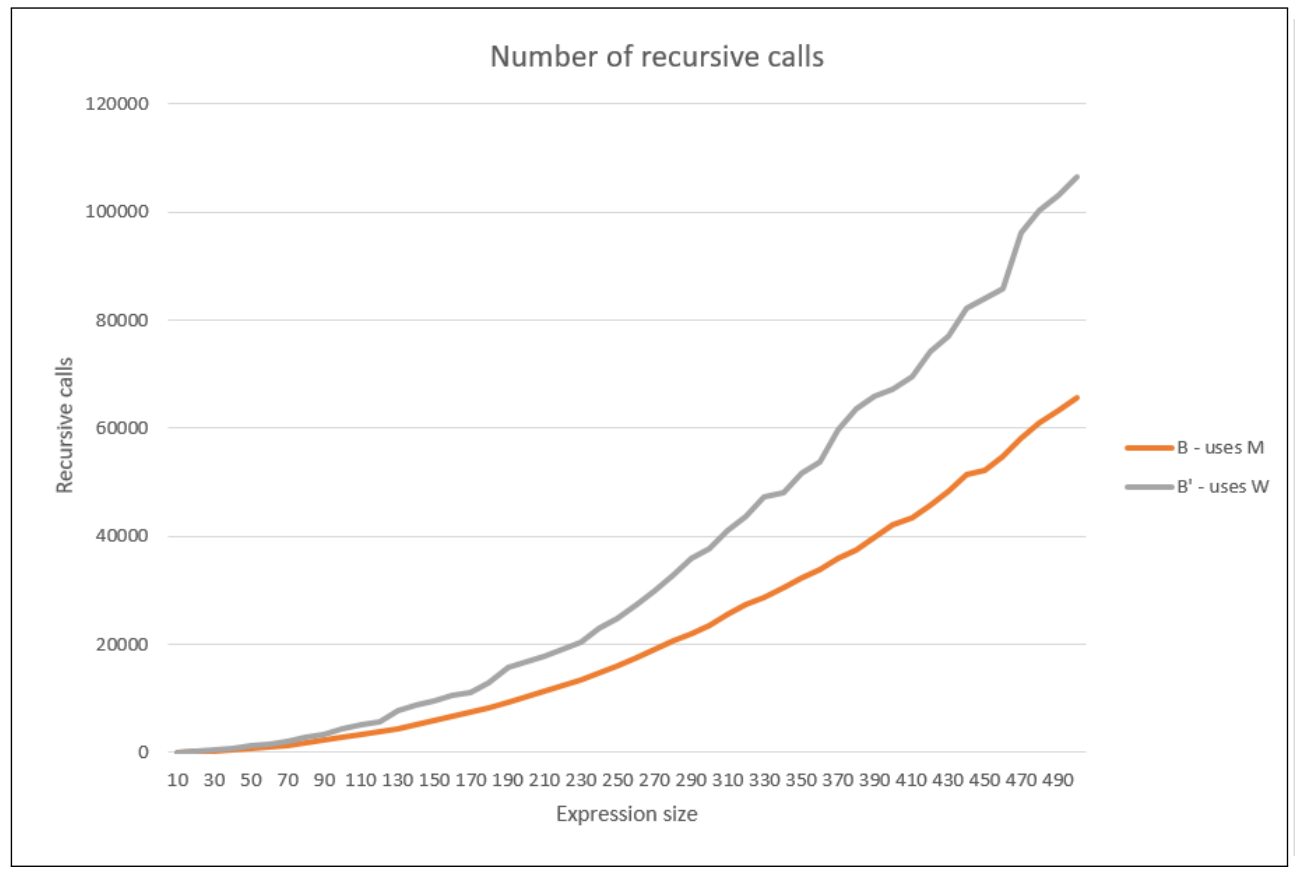

Figure 5: Number of recursive calls for a set expression size

with a random expression generator to test the two approaches discussed in this paper.

The size of an expression e is:

$$
\operatorname{size}(e)= \begin{cases}1, & \text { if } e \equiv x \\ 1+\operatorname{size}(f)+\operatorname{size}(g), & \text { if } e \equiv(f @ g) \\ 1+\operatorname{size}(f), & \text { if } e \equiv(\lambda x . f)\end{cases}
$$

We will generate random untyped lambda expressions and calculate the total number recursive calls made by the inference algorithms depending on the size of the input. We will be using and algorithm developed by Jue Wang [12]. This algorithm generates random $\lambda$-terms of a given size, assuming a uniform distribution over all terms of a given size.

These calculations will be run with empty starting type environments. For each size we will generate a hundred expressions, and take the average of the visited nodes/recursive steps made by the algorithms. 
We can see that the numbers of recursive calls are super linear in both cases but there is a noticeable difference in the multiplier. This data corresponds only to the number of recursive calls made by $\underline{\mathcal{M}}$ and $\underline{\mathcal{W}}$. It is important to keep in mind that this doesn't take the cost of unification into consideration. This is why we have also included the time spent by both algorithms in the following figure.

\begin{tabular}{|l||l|l|}
\hline \multicolumn{3}{|c|}{ Mean execution time } \\
\hline Size & $\mathcal{B}$ & $\mathcal{B}^{\prime}$ \\
\hline 100 & $1.09 \mathrm{~ms}$ & $1.91 \mathrm{~ms}$ \\
250 & $3.93 \mathrm{~ms}$ & $6.84 \mathrm{~ms}$ \\
500 & $88.4 \mathrm{~ms}$ & $113 \mathrm{~ms}$ \\
1000 & $1.10 \mathrm{~s}$ & $1.58 \mathrm{~s}$ \\
\hline
\end{tabular}

Both $\mathcal{B}$ and $\mathcal{B}^{\prime}$ were implemented in Haskell. The unification algorithm and the definitions describing the type system and the expressions are common between the two algorithms. The code for generating $\lambda$-terms is also available. ${ }^{2}$ To facilitate exception throwing and the calls for new type variables, we used a monad stack in the type inference algorithms.

type TyVar $=$ Int

data Type $=$ TBase $[$ Occurrence $]$

| TArrow Type Type [Occurrence]

| TVar TyVar

| TUntyped

type $\mathrm{TI} \mathrm{a}=$ ExceptT [Occurrence] (State TyVar) a

The core types and definitions are located in Common, $\mathcal{B}$ and $\mathcal{B}^{\prime}$ are found in $A l g o M$ and $A l g o W$ respectively. A basic parser is provided in Parser for interactive testing. Testing includes the functions and generators used for random testing.

\section{Conclusion}

Binding time analysis and partial evaluation are well-researched fields. Possible advances can only mostly be done to better efficiency and support a wider

\footnotetext{
${ }^{2}$ Link to publicly available repository: https://github.com/szokolimatyas/Type-Inferencefor-BTA
} 
range of language features. This paper proposes some modest improvements to an existing algorithm without great changes. These improvements speed up the algorithm, but not by orders of magnitude, still having a super-linear complexity.

It is not possible to achieve linearity for these types of "restarting" inference algorithms, as in the worst case - which means every operator needs to be underlined - for an expression with a size $s$ the complexity is $s * \theta(k)$. One run of the algorithm tries to find the next operator that needs to be underlined, which $(\theta(k))$ is almost linear in practice. If we used the Hindley-Milner type system, the inference algorithm would mostly have a polynomial complexity. However, there are some pathological cases where it is non-linear [14]. Intuitively, we run the algorithm $s$ times, with the time complexity proportional to $s$ each time.

It would be remiss if we did not mention the work of Henglein [5], who took a more constraint-oriented approach based on Gomard, proposing a near linear time solution to binding time analysis.

There are some more efficient algorithms provided for BTA as mentioned in the related works, but this direction still can be more thoroughly explored. Neither was the use of type inference for detecting type conflicts in untyped programs deeply examined formerly.

We feel that this algorithm has legitimacy, because it can be applied to a wide range of problems, promises good prospects for further advancement, and is easily derived from existing approaches, providing an easy to understand solution to binding time analysis and analyzing dynamically typed programs.

\section{Future work}

Type inference algorithms have been developed in more descriptive type systems and we think that the same type of modifications could also be applied there to produce similar results. Namely, the two algorithms described in this paper can also be used in the Hindley-Milner system, even with letpolymorphism. One hindrance is that in some cases inference is not decidable [13]. This can cause problems if we wanted to use it for better error messages in statically typed languages. Binding time analysis, however, could fare better, as we don't differentiate between type constants, so we could avoid some of the ambiguity. 
We have not implemented the proposed changes by Gomard: the addition of lifting and reporting more than one occurrences is possible avenue for improvement. It is also trivial to change $\underline{\mathcal{M}}$ to support let-polymorphism.

\section{Acknowledgements}

The project has been supported by the European Union, co-financed by the European Social Fund (EFOP-3.6.3-VEKOP-16-2017-00002).

\section{References}

[1] K. Asai, Toward introducing binding-time analysis to MetaOCaml, Proceedings of the 2016 ACM SIGPLAN Workshop on Partial Evaluation and Program Manipulation, St. Petersburg, FL, USA, 2016, pp. 97-102. $\Rightarrow 233$

[2] K. Claessen, J. Hughes, Quickcheck: A lightweight tool for random testing of Haskell programs, SIGPLAN 35, 9 (2000) 268-279. $\Rightarrow 245$

[3] C. Gomard, N. Jones, A partial evaluator for the untyped lambda calculus, Journal of Functional Programming 1, 1 (1991) 21-69. $\Rightarrow 233$

[4] C. K. Gomard, Partial type inference for untyped functional programs, Proceedings of the 1990 ACM Conference on LISP and Functional Programming, Nice, France, 1990, pp. 282-287. $\Rightarrow 232,234,235$

[5] F. Henglein, Efficient type inference for higher-order binding-time analysis, Conference on Functional Programming Languages and Computer Architecture, Lecture Notes in Computer Science 523 (1991) 448-472. $\Rightarrow 248$

[6] O. Lee, K. Yi, Proofs about a folklore let-polymorphic type inference algorithm, ACM Trans. Program. Lang. Syst., 20, 4 (1998) 707-723. $\Rightarrow 235,237,240$

[7] T. Lindahl, K. Sagonas, Practical Type Inference Based on Success Typings, Proc. 8th ACM SIGPLAN International Conference on Principles and Practice of Declarative Programming, Venice, Italy, 2006, pp. 167-178. $\Rightarrow 234$

[8] R. Milner, L. Damas, Principal type-scheme for functional programs, Proc. 9th ACM Symposium on Principles of Programming Languages New York, USA, 1982 , pp. $207-212 . \Rightarrow 240$

[9] T. Murakami, Z. Hu, K. Kakehi, M. Takeichi, An efficient staging algorithm for binding-time analysis, International Symposium on Logic-Based Program Synthesis and Transformation, Lecture Notes in Computer Science 3018 (2003) pp. $106-107 . \Rightarrow 233$

[10] H. R. Nielson, F. Nielson, Automatic binding time analysis for a typed lambdacalculus (extended abstract), Proc. 15th ACM SIGPLAN-SIGACT symposium on Principles of programming languages San Diego, California, USA, 1988, pp. $98-106 . \Rightarrow 233$ 
[11] T. Sheard, N. Linger, Search-based binding time analysis using type-directed pruning, Proceedings of the ASIAN Symposium on Partial Evaluation and Semantics-Based Program Manipulation, Aizu, Japan, 2002, pp. 20--31. $\Rightarrow 233$

[12] J. Wang, Generating random lambda calculus terms, Semantic Scholar, 2005. https://www.semanticscholar.org $\Rightarrow 246$

[13] J. B. Wells, Typability and type checking in system $\mathrm{F}$ are equivalent and undecidable, Annals of Pure and Applied Logic, 98, 1 (1999) $111-156 . \Rightarrow 248$

[14] H. G. Mairson, Deciding ML typability is complete for deterministic exponential time, Proc. 17th ACM SIGPLAN-SIGACT symposium on Principles of programming languages, New York, NY, United States, 1989, pp. 382-401. $\Rightarrow 248$

[15] B. C. Pierce, Advanced Topics in Types and Programming Languages, MIT Press, Cambridge, MA, 2004. $\Rightarrow 233$

[16] B. C. Pierce, Types and Programming Languages, MIT Press, Cambridge, MA, 2002. $\Rightarrow 233$ 